\title{
Thermal transmission of the fluent water Chuang zhao
}

School of North China Electric Power University, Baoding071000, China; 15733228611@163.com

Keywords: Open system, numeric calculation, conservation of energy.

\begin{abstract}
We study the thermal transmission of the fluent water whit steady velocity. First we divide the water into lots of little grids in two-dimensional space and study the temperature distribution of the fluent water. To make accurate numerical calculation, we then regard the grid in fluent water as an open system and establish discretions equations of the different situation. Finally, we calculate relevant date by the iterative method of Gauss-Seidel and get the temperature distribution in space.
\end{abstract}

\section{Introduction}

The condition of the motionless water can be calculate by the traditional numerical calculation, but the thermal transmission of fluent water is too complex to the traditional calculation[1]. In this article we extend traditional model and assume that each grid is an open system, and we analyze the thermal transmission of the grid in open system at the base of the laws of thermodynamics.

\section{Analysis of the thermal transmission}

To make clear the thermal transmission of the fluent water, we divide the water into myriad little grids and assume that every grid is an open system[2], then we analyze the inner grids. Since the hot water is flowing at a constant speed, we can study the thermal transmission of the fluent water referring the conservation of the fluent water.

We first analyze the inner grids in new two-dimensional, the fluency of the water in each grid is showed in flowing picture:

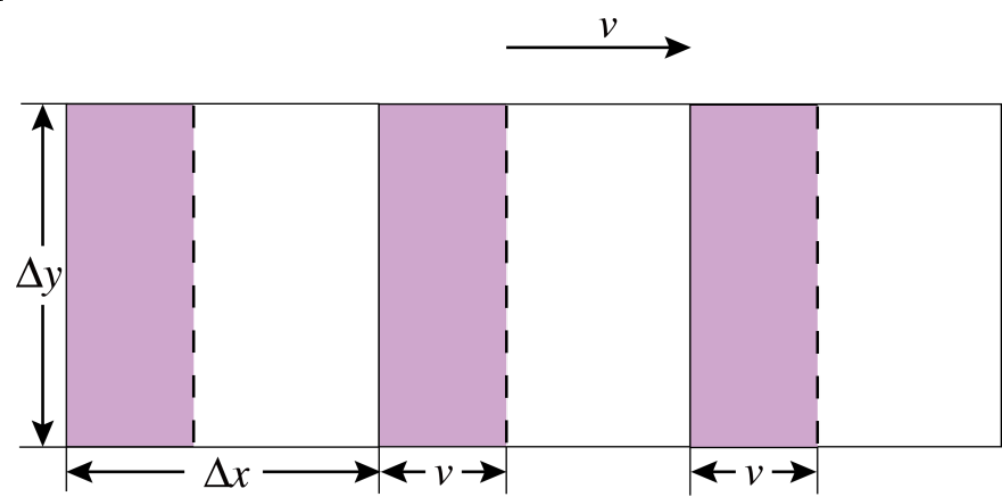

Fig. 1 The situation that water flowing in the open system

As is showed on Fig. 1, we know that much thermal is transferred from one grid to its next grid. Since the velocity of the water is stable, we can calculate the numeric of the thermal transferred by the adjacent grids with the temperature of the border[3]. With the basis of the laws of thermodynamics, we could analyze the thermal transmission in the open system: 


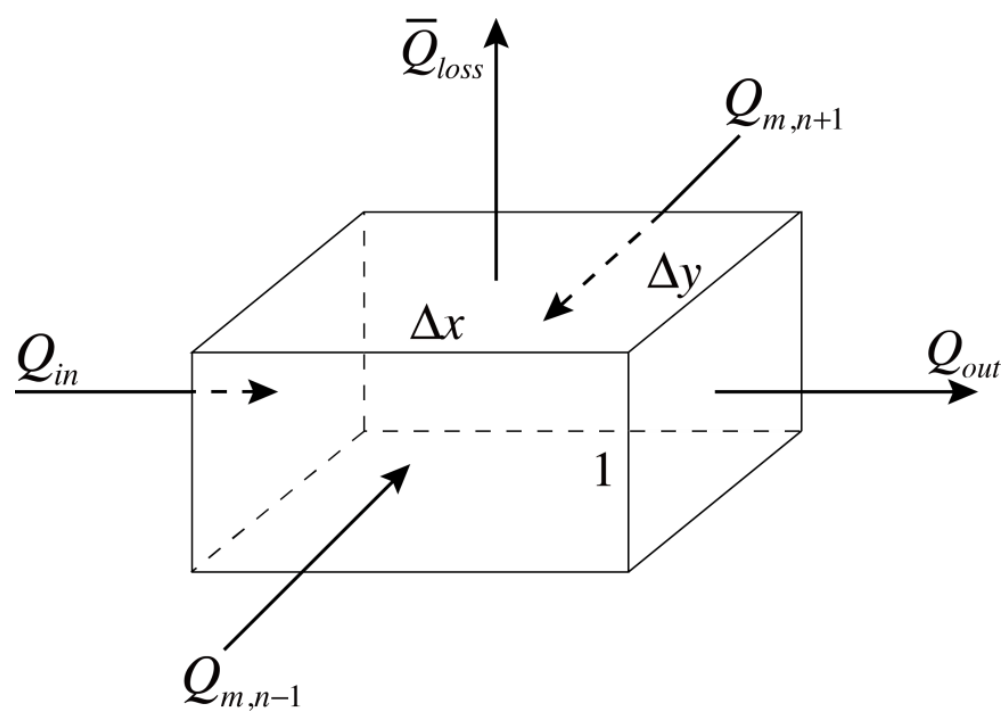

Fig. 2 Thermal transmission on the open system

As we can see, the thermal conduction of the new grid with the grids along the width and the evaporation thermal loss averaged to every grid has no change. And the thermal conduction along the length is neglected since the water is fluent on this direction.

What's more, there is considerable heat transferred by the flow of the water from the previous grid. In a similar way, the grid loss some heat with the drainage to the next grid.

\section{Numerical Calculation}

Table 1 Symbol table

\begin{tabular}{|c|c|c|}
\hline Symbol & Meaning & Value/Units \\
\hline $\mathrm{Q}_{\text {in }}$ & The extra heat from the outside world & $\mathrm{kJ}$ \\
\hline$Q^{\prime} m, n$ & $\begin{array}{l}\text { the extra heat from the flowing water of grid }(m, n) \\
\text { from grid }(m-1, n) \text {. }\end{array}$ & $\mathrm{kJ}$ \\
\hline$\lambda$ & The ability of conducting heat & $\mathrm{W} /(\mathrm{m} \cdot \mathrm{K})$ \\
\hline $\mathrm{t}_{0}$ & The temperature of environment & ${ }^{\circ} \mathrm{C}$ \\
\hline $\mathrm{t}_{\mathrm{m}, \mathrm{n}}$ & The temperature of the grid $(\mathrm{m}, \mathrm{n})$ & ${ }^{\circ} \mathrm{C}$ \\
\hline$\rho$ & The density of the water & $\mathrm{kg} / \mathrm{m}^{3}$ \\
\hline c & Specific heat capacity of the water & $\mathrm{J} /(\mathrm{kg} \cdot \mathrm{K})$ \\
\hline
\end{tabular}

To make sure the numeric of the $Q_{i n}$, we assume that the temperature of the water is constant when flowing, thus we can know that the heat transferred by the fluent water is determined by the temperature of the previous grid[4].

$$
Q_{m, n}^{\prime}=c \rho V\left(t_{m-1, n}-t_{m, n}\right)=c \rho \Delta x \Delta y\left(t_{m-1, n}-t_{m, n}\right)
$$

Then we can get the whole change of the heat from the flowing water ingrid $(m, n)$ :

$$
\Delta Q=Q_{m, n}^{\prime}-Q_{m+1, n}^{\prime}=c \rho \Delta x \Delta y\left(t_{m-1, n}+t_{m+1, n}-2 t_{m, n}\right)
$$

Since the temperature distribution is stable, the temperature of every grid doesn't change with time, we can establish the following equation of the inner grid: 


$$
\begin{aligned}
& \lambda \Delta x \frac{t_{m, n-1}-t_{m, n}}{\Delta y}+\lambda \Delta x \frac{t_{m, n+1}-t_{m, n}}{\Delta y}+c \rho \Delta x \Delta y\left(t_{m-1, n}+t_{m+1, n}-t_{m, n}\right) \\
& -k \alpha \Delta x \Delta y\left(t_{m, n}-t_{0}\right)=0
\end{aligned}
$$

The temperature of the grids on boundary can be calculated by :

$$
\begin{aligned}
& \lambda \Delta x \frac{t_{m, n-1}-t_{m, n}}{\Delta y}+\lambda \Delta x \frac{t_{m, n+1}-t_{m, n}}{\Delta y}+c \rho \Delta x \Delta y\left(t_{m-1, n}+t_{m+1, n}-t_{m, n}\right) \\
& -\frac{k \alpha \Delta x \Delta y\left(t_{m, n}-t_{0}\right)}{2}=0
\end{aligned}
$$

And the temperature of the grids at corner can be calculated by:

$$
\begin{aligned}
& \lambda \Delta x \frac{t_{m, n-1}-t_{m, n}}{\Delta y}+\lambda \Delta x \frac{t_{m, n+1}-t_{m, n}}{\Delta y}+\frac{c \rho \Delta x \Delta y\left(t_{m-1, n}+t_{m+1, n}-t_{m, n}\right)}{2} \\
& -\frac{k \alpha \Delta x \Delta y\left(t_{m, n}-t_{0}\right)}{4}=0
\end{aligned}
$$

To get the numeric needed more quickly, we also calculate our equations by the iterative method of Gauss-Seidel.

Then we simplify the (3) (4) (5)and get the expression about $t_{m, n}^{i+1}, t_{m, n}^{i}$ and other factors of the three typical grids, and all the grids of the plane could be expressed at the basis of the equations above.

Before our calculation, we should check the coefficient of the $t_{m, n}^{i}$ about the $t_{m, n}^{i+1}$, and the numeric of the coefficient should be greater than or the same as 0 . It is because the discretion equations we establish is explicit scheme, if the coefficient of the $t_{m, n}^{i}$ is less than 0 , the equation will violate the law of thermodynamics. Combined with this qualification and the simplification of calculation, we have $\Delta \tau=60$ s.

To get the numeric needed more quickly, we determine to calculate relevant date by the iterative method of Gauss-Seidel[5]. To get the temperature distribution of the water in space and time, we should set suitable boundary conditions.

Thus we can make sure the boundary conditions of the model, and with the relevant date we can get the detailed temperature of every grid at any time with the help of the computer and we can make intuitional description of the temperature distribution of the water in space and time.

\section{Summary}

If we seem the fluent water as the open system which follows the laws of thermodynamics, we can make good numeric calculation and get the distribution of the water in space and time.

\section{References}

[1]Bowen I S, The ratio of heat losses by conduction and by evaporation from any water surface[J], Physical review, 1926, 27(6): 779.

[2] Patankar S. Numerical heat transfer and fluid flow[M]. CRC Press, 1980.

[3] Baker Jr G A, Oliphant T A. An implicit, numerical method for solving the two-dimensional heat equation[R]. Los Alamos Scientific Lab., N. Mex., 1958.

[4]Douglas J, Gunn J E. Two high-order correct difference analogues for the equation of multidimensional heat flow[J]. Mathematics of Computation, 1963, 17(81): 71-80. 
[5] Lorenzi D L, Porter R W. Simplified Analysis of Surface Energy Exchange from Heated Bodies of Water[J]. Advances in Heat and Mass Transfer at Air-Water Interfaces, Edited by S. Sengupta, ASME, 1978: 93-101. 